\title{
Signatures of magnetic reconnection during the evolutionary phases of a prominence eruption and associated X1.8 flare
}

\author{
Bhuwan Joshi $^{1}$, Upendra Kushwaha ${ }^{1}$, KyungSuk Cho ${ }^{2}$ and \\ Astrid Veronig ${ }^{3}$ \\ ${ }^{1}$ Udaipur Solar Observatory, Physical Research Laboratory, Udaipur 313004, India \\ ${ }^{2}$ Korea Astronomy and Space Science Institute, Daejeon 305-348, Korea \\ ${ }^{3}$ IGAM/Institute of Physics, University of Graz, Universitätsplatz 5, A-8010 Graz, Austria
}

\begin{abstract}
In this paper, we present RHESSI and TRACE observations of multiple flare activity that occurred in the active region NOAA 10656 on 2004 August 18. Out of four successive flares, there were three events of class-C while the final event was a major X1.8 solar eruptive flare. During localized C-class flares, the filament undergoes slow yet crucial morphological evolution. The filament eruption is accompanied with an X1.8 flare during which multiple HXR bursts are observed up to $100-300 \mathrm{keV}$ energies. From the location, timing, strength, and spectrum of HXR emission, we conclude that the prominence eruption is driven by the distinct events of magnetic reconnection occurring in the current sheet below the erupting prominence. These multi-wavelength observations also provide evidence for tether-cutting reconnection as the triggering mechanism for filament eruption and associated X-class flare.
\end{abstract}

Keywords. flares, filaments, X-rays, magnetic reconnection.

\section{Introduction}

The association of eruptions of solar prominences with coronal mass ejections and solar flares is well known. It is widely accepted that the fundamental processes responsible for these phenomena are closely related and of magnetic origin. Multi-wavelength measurements are crucial to infer the characteristics of physical processes responsible for impulsive energy release and eruptions (Joshi et al. 2012). In this paper, we report RHESSI and TRACE observations of the eruption of an active region filament (Cho et al. 2009, Joshi et al. 2013) occurred in NOAA 10656 on 2004 August 18. We present observations of four flares (there were three flares of class $\mathrm{C}$ while the last events was an X1.8 eruptive flare) that occur during the evolutionary phases of the filament toward its eruption (Fig. 1).

\section{Observations and results}

1. The pre-eruption phase is characterized by three localized flares (i.e., events I, II and III) which are associated with heating, activation, and rise of the filament (Fig. 2(a)-(d)). During the first event, we observed plasmoid eruption (Fig. 1(a)) which was followed by the rise of filament (Fig. 2(b)). Events II and III occurred close to the footpoints of the rising filament. The pre-eruption flares and subsequent changes in the morphology of the prominence provide evidence for the tether-cutting mechanism for solar eruptions (Moore \& Roumeliotis 1992).

2. The filament eruption was accompanied by an X1.8 flare (Fig. 2(e)-(f)) during which multiple HXR bursts are observed up to 100-300 keV energies. We observed a bright and 


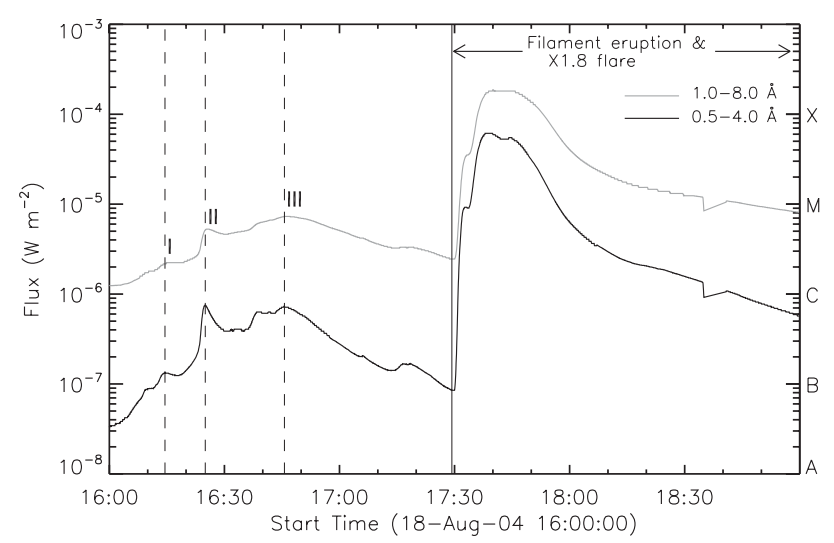

Figure 1. GOES soft X-ray time profiles in the $0.5-4$ and $1-8 \AA$ wavelength bands. The vertical solid line differentiates the pre-eruption phase from the eruptive X1.8 flare. Three C-class flares observed before the prominence eruption are denoted as I, II, and III.
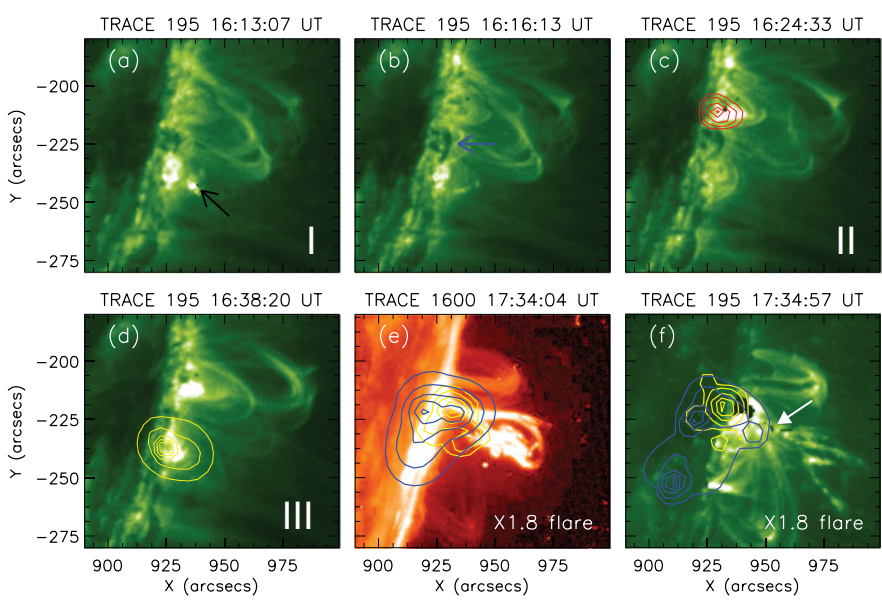

TRACE 1600 17:34:04 UT
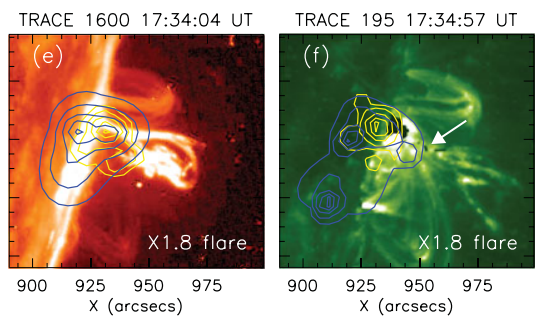

Figure 2. TRACE observations of pre-eruption events (I, II, and III; panels (a)-(d)) and X1.8 class flare associated with prominence eruption (panels (e)-(f)). Arrow in panel (b) indicates the rise of prominence after event I. Contours represent RHESSI PIXON images in $12-25 \mathrm{keV}$ (yellow), 25-50 keV (red), and 50-100 keV (blue) energy bands.

extended coronal source simultaneously in EUV and 50-100 keV HXR images underneath the expanding filament which provides strong evidence for the ongoing magnetic reconnection. This phase is accompanied with very high plasma temperatures of $\sim 31$ MK which is followed by the detachment of the prominence from the solar source region.

\section{References}

Cho, K.-S., Lee, J., Bong, S.-C., Kim, Y.-H., Joshi, B., \& Park, Y.-D. 2009, ApJ, 703, 1

Joshi, B., Veronig, A., Manoharan, P. K., \& Somov, B. V. 2012, in Astrophysics and Space

Science Proceedings, Vol. 33, Multi-scale Dynamical Processes in Space and Astrophysical

Plasmas, ed. M. P. Leubner \& Z. Vörös (Springer-Verlag Berlin Heidelberg), 29

Joshi, B., Kushwaha, U., Cho, K.-S., \& Veronig, A. M., ApJ, 771, 1

Moore, R. L. \& Roumeliotis, G. 1992, in Lecture Notes in Physics, Berlin Springer Verlag, Vol. 399, IAU Colloq. 133: Eruptive Solar Flares, ed. Z. Svestka, B. V. Jackson, \& M. E. Machado, 69 